\title{
Performance of Endobronchial
}

\section{Ultrasound-Guided Transbronchial Needle Aspiration for the Diagnosis of Isolated Mediastinal and Hilar Lymphadenopathy}

\author{
Chung-Chun Tyan ${ }^{a, b}$ Tiago Machuca ${ }^{a}$ Kasia Czarnecka ${ }^{a}$ b Hyang Mi Ko ${ }^{b}$ \\ Gilda da Cunha Santos ${ }^{b}$ Scott L. Boerner ${ }^{b} \quad$ Andrew Pierre $^{a} \quad$ Marcelo Cypel $^{a}$ \\ Tom Waddella Gail Darling ${ }^{a} \quad$ Marc de Perrot $^{a} \quad$ Shaf Keshavjee $^{a}$ \\ William Geddie $^{b}$ Kazuhiro Yasufuku ${ }^{a}$ \\ ${ }^{a}$ Division of Thoracic Surgery, ${ }^{b}$ Division of Respirology, and ${ }^{\mathrm{C}}$ Department of Laboratory Medicine and Pathobiology, \\ University Health Network, University of Toronto, Toronto, ON, Canada
}

\section{Keywords}

Mediastinal adenopathy - Endobronchial ultrasound .

Bronchoscopy · Sarcoidosis · Lymphoproliferative disease

\begin{abstract}
Background: Although many studies have assessed the diagnostic utility of endobronchial ultrasound-guided transbronchial needle aspiration (EBUS-TBNA) in the context of a specific disease, few studies have assessed the overall diagnostic yield, sensitivity, and negative predictive value in patients with isolated mediastinal and hilar lymphadenopathy (IMHL). Objective: We evaluated the performance of EBUSTBNA for diagnosing IMHL in a population with a high prevalence of concurrent or preexisting non-pulmonary malignancy. Methods: A retrospective chart review of patients who underwent EBUS-TBNA from October 2008 to April 2014 was performed to identify patients with IMHL. Patients with known or suspected primary pulmonary malignancy were excluded. When available, EBUS-TBNA results were cross-referenced with further diagnostic investigation or clinical diagnosis based on follow-up. Results: EBUS-TBNA
\end{abstract}

\section{KARGER}

(C) 2017 S. Karger AG, Basel

E-Mail karger@karger.com

www.karger.com/res was used to sample 765 lymph nodes from 350 patients. One hundred and fourteen (33.3\%) patients had a concurrent or preexisting non-pulmonary malignancy. The overall yield of EBUS-TBNA for specific diagnosis was 300/350 (86\%). The diagnostic yield for sarcoidosis, lymphoproliferative disease, metastatic lymphadenopathy from extrathoracic malignancy, and necrotizing granuloma was 123/149 (83\%), 27/33 (82\%), 20/25 (80\%), and 13/19 (68\%), respectively. Amongst 50 patients with non-diagnostic EBUS-TBNA, 25 yielded an insufficient sample and another 25 yielded only benign lymphoid material which was not representative of the underlying pathology. Overall, EBUS-TBNA had a sensitivity of $89 \%$, a diagnostic yield of $86 \%$, and a negative predictive value of $79 \%$. Conclusion: For patients with isolated hilar or mediastinal lymphadenopathy and a high background prevalence of concurrent and preexisting non-pulmonary malignancy, EBUS-TBNA is a reliable first-line diagnostic investigation.

(c) 2017 S. Karger AG, Basel

This study was presented at the 19th WCBIP World Congress, May 9, 2016, Florence, Italy. 


\section{Background}

Patients with isolated mediastinal and hilar lymphadenopathy (IMHL) pose a diagnostic challenge. Non-specific signs such as fever, weight loss, and decreased energy are shared by common IMHL differentials such as sarcoidosis, tuberculosis, lymphoma, and malignant carcinoma. Adding to the diagnostic dilemma, reactive lymphocytosis is associated with prevalent chronic diseases such as chronic obstructive lung disease [1], interstitial lung disease [2], and congestive heart failure [3, 4]. Therefore, patients with IMHL often require an invasive procedure to obtain tissue for cytohistologic and microbiologic assessment.

Endobronchial ultrasound-guided transbronchial needle aspiration (EBUS-TBNA) is an established approach for mediastinal lymph node sampling during staging of patients with lung cancer. Given the success obtained by EBUS-TBNA for lung cancer staging [5], it is increasingly utilized in the investigation of IMHL. Current studies investigating the role of EBUS-TBNA in IMHL demonstrate a consistent diagnostic yield for nonnecrotizing granuloma [6] and metastatic lymphadenopathy from extrathoracic malignancy [7]. However, studies investigating the diagnostic yield of lymphoma by EBUS-TBNA reported a range from 50 to $90 \%$ [8].

Although many studies have assessed the diagnostic utility of EBUS-TBNA in the context of a specific disease, only a few have assessed the overall diagnostic yield, sensitivity, and negative predictive value (NPV) in patients with IMHL. In particular, the NPV of EBUS in IHML has been reported to be around mid-20-40\% $[9,10]$ until recent data published by Evison et al. [11]. In this study, we evaluate EBUS-TBNA for diagnosing IMHL in a population with a high prevalence of concurrent or preexisting non-pulmonary malignancy.

\section{Material and Methods}

\section{Patients}

The EBUS-TBNA database from the Toronto General Hospital, dating from October 2008 to April 2014, was reviewed. Patients with IMHL in the absence of a known or suspected primary lung cancer were included in the study. This study was conducted in accordance with the amended Declaration of Helsinki. University Health Network Research Ethics Board approval was obtained for this study. The Research Ethics Board identifying number is 14-7383.

EBUS-TBNA Procedure

The EBUS-TBNA was performed with a convex probe endobronchial ultrasound bronchoscope (BF-UC180F, Olympus, To- kyo, Japan) and connected to a dedicated ultrasound scanner (EUY0005; Olympus) with color Doppler flow imaging for the detection of blood vessels. The dedicated 22-G TBNA needle (NA201SX-4022; Olympus) was used to perform EBUS-TBNA. Once the needle was advanced into the mass, the internal stylet was used to clear the tip of the needle. The 20-mL VacLoc (Merit Medical Systems, Inc., South Jordan, UT, USA) syringe was used to generate suction during the majority of passes.

\section{Rapid On-Site Evaluation}

We aimed to select all cases with a clinical suspicion for lymphoproliferative disease and/or granulomatous disease to have rapid on-site evaluation (ROSE). However, due to limitation in human resource, not all cases with the specified clinical indication had ROSE in attendance.

\section{Sample Process}

For cases performed without ROSE, the samples collected from the TBNA needle were flushed into Cytolyt ${ }^{\circledR}$ fixative with an airfilled 20 -mL syringe or $5 \mathrm{~mL}$ sterile normal saline when infectious etiology was suspected.

For cases performed with ROSE, the stylet was used to push out the sample in the needle for on-site evaluation. The material was used to prepare wet-fixed and air-dried smears. A representative air-dried smear was stained with a rapid Romanowsky-type stain (Field's stain). Once the cytopathologist deemed the specimen adequate, appropriate ancillary studies were initiated. If adequate tissue was not identified by on-site cytology after 5 passes, the procedure was terminated.

\section{Ancillary Testing}

The specimens were triaged to appropriate ancillary studies according to the preliminary cytomorphologic diagnosis. When infection was suspected or the slide demonstrated granulomas, an aliquot from needle rinse was sent for mycobacterial and fungal culture. If the slides demonstrated carcinoma on ROSE, the needle rinse was processed to produce formalin-fixed, paraffin-embedded cell blocks. While cytomorphological assessment demonstrated large-cell lymphoma or small lymphoid proliferation that suggested lymphoma, the specimens were submitted for flow cytometry.

\section{Data Collected}

Baseline patient and nodal characteristics were collected. Based on clinical presentation, a preprocedural diagnosis was made and used to facilitate the utilization of ROSE. The final diagnosis was based on EBUS-TBNA or further invasive investigation. All patients' charts were reviewed up to October 2015 to ensure that the pathologic diagnosis was consistent with the clinical course. In cases where the clinical course did not agree with the pathologic diagnosis, all data were reviewed and used to formulate the most likely diagnosis. If patients were followed up in the community without on-site information available to review by electronic medical record, a letter was sent to the referring physician to obtain data on further investigations and final diagnosis.

\section{Statistical Analysis}

Continuous variables such as the patient's age and lesion size were summarized as means and standard deviations. Categorical variables such as sex, lymph node station, diagnosis, and further 
Table 1. Baseline characteristics and final diagnosis

\begin{tabular}{lc}
\hline Total patients & $350(100)$ \\
Mean age \pm SD, years & $57 \pm 14$ \\
Male, female & $198,152(57,43)$ \\
Known concurrent or preexisting malignancy & $114(33)$ \\
Malignant diagnosis & $75(21)$ \\
$\quad$ Lymphoma & $33(9)$ \\
$\quad$ Metastatic lymphadenopathy from extrathoracic malignancy & $25(7)$ \\
$\quad$ Lung cancer & $16(5)$ \\
$\quad$ Posttransplant lymphoproliferative disorder & $1(0.3)$ \\
Benign diagnosis & $275(79)$ \\
$\quad$ Sarcoidosis & $149(43)$ \\
$\quad$ Reactive lymphocytosis & $101(29)$ \\
$\quad$ Necrotizing granuloma including TB, NTM, and fungal infection & $19(5)$ \\
$\quad$ Others* & $6(2)$ \\
\hline \multicolumn{2}{l}{ Figures are $n(\%)$ unless indicated otherwise. SD, standard deviation; NSCLC, non-small-cell lung cancer; TB, } \\
tuberculosis; NTM, non-tuberculous mycobacterium. * Others include bronchogenic cysts $(n=2)$, histiocytosis \\
$(n=1)$, mastocytosis $(n=1)$, amyloidosis $(n=1)$, and silicosis $(n=1)$.
\end{tabular}

interventions were expressed as frequencies and proportions. Patients with a final diagnosis of reactive lymphocytosis were defined as true negative, while patients initially diagnosed with reactive lymphocytosis on EBUS-TBNA but later proven to have an alternative diagnosis by means of pathology or clinical course were defined as false negative. EBUS procedures that yielded insufficient samples were not included in the calculation of sensitivity and NPV but were included in the calculation of the diagnostic rate.

\section{Results}

Three hundred and fifty patients were included in the study (Table 1). In total, 765 lymph nodes were biopsied (Table 2). EBUS-TBNA established a diagnosis for 300 patients (86\%; Table 3). Twenty-five patients (7\%) had insufficient sampling from EBUS-TBNA and were considered technically unsuccessful procedures. Twenty-one patients were not followed up in our institution and we were not able to obtain clinical information beyond the EBUS procedure. All other patients were followed for a minimal of 1 year and beyond.

Within the benign disease patient category, sarcoidosis was the most prevalent diagnosis $(n=149)$. When transbronchial forceps biopsy was performed on 21 patients with mucosal changes consistent with sarcoidosis, 7 additional patients were diagnosed with sarcoidosis. Twenty-eight patients diagnosed with sarcoidosis had a previously treated or current extrapulmonary malignancy (Fig. 1).

Performance of EBUS-TBNA in

Mediastinal Disease
Table 2. Frequency of mediastinal and hilar lymph nodes sampled

\begin{tabular}{lcll}
\hline $\begin{array}{l}\text { Lymph } \\
\text { node } \\
\text { station }\end{array}$ & $n(\%)$ & $\begin{array}{l}\text { Avg. horizontal } \\
\text { axis }^{\mathrm{a}}( \pm \mathrm{SD}), \mathrm{mm}\end{array}$ & $\begin{array}{l}\text { Avg. vertical axis } \\
( \pm \mathrm{SD}), \mathrm{mm}\end{array}$ \\
\hline All & $765(100)$ & $17(8)$ & $13(9)$ \\
$2 \mathrm{R}$ & $16(2)$ & $15(6)$ & $10(7)$ \\
$4 \mathrm{R}$ & $219(29)$ & $15(5)$ & $11(5)$ \\
$4 \mathrm{~L}$ & $41(4)$ & $14(13)$ & $10(10)$ \\
7 & $265(35)$ & $20(9)$ & $17(13)$ \\
$10 \mathrm{R}$ & $10(1)$ & $15(4)$ & $12(3)$ \\
$10 \mathrm{~L}$ & $4(1)$ & $20(5)$ & $17(2)$ \\
$11 \mathrm{R}$ & $88(12)$ & $15(7)$ & $12(6)$ \\
$11 \mathrm{~L}$ & $105(14)$ & $14(4)$ & $11(3)$ \\
$12 \mathrm{R}$ & $17(2)$ & $15(6)$ & $13(5)$ \\
\hline
\end{tabular}

SD, standard deviation; Avg., average. ${ }^{\text {a }}$ Maximal length of the lymph node that is parallel to the examined airway. ${ }^{\mathrm{b}}$ Maximal length of the lymph node that is vertical to the examined airway.

The remaining category of the benign diagnosis consisted of reactive lymphocytosis $(n=101)$, necrotizing granulomatous diseases $(n=19)$, and others $(n=6)$. Of those diagnosed with necrotizing granuloma, 10 patients were diagnosed with either tuberculosis or non-tuberculous mycobacteria and 3 patients were diagnosed with fungal infection.

Lymphoproliferative disease was established in 33 patients: 9 Hodgkin lymphoma and 24 non-Hodgkin lymphoma. Twenty-seven patients were diagnosed by EBUS- 
Fig. 1. Final diagnoses in patients with concurrent or preexisting malignancy. EBUS-TBNA, endobronchial ultrasound-guided transbronchial needle aspiration. Bold numbers are the number of patients overlapped by patients with a history of intrathoracic lymphadenopathy with concurrent or preexisting malignancy. * Non-pulmonary malignant lymphadenopathy patients include patients with metastatic lymphadenopathy from extrathoracic malignancy and lymphoma.

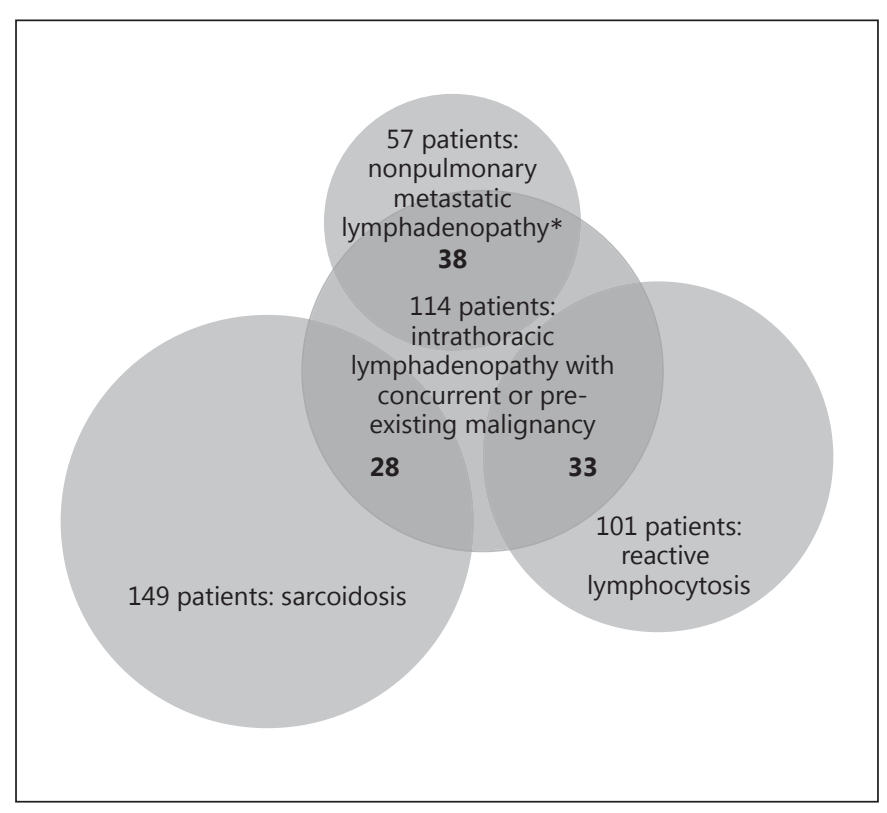

Table 3. Diagnostic rate of EBUS-TBNA, further invasive investigation performed after EBUS-TBNA and utilization of ROSE

\begin{tabular}{|c|c|c|c|}
\hline Diagnosis & $\begin{array}{l}\text { Diagnostic by } \\
\text { EBUS-TBNA, } \\
n / N(\%)\end{array}$ & $\begin{array}{l}\text { Diagnostic } \\
\text { with ROSE, } \\
n / N(\%)\end{array}$ & $\begin{array}{l}\text { Further invasive } \\
\text { intervention, } \\
n / N(\%)\end{array}$ \\
\hline Malignant diagnosis & $63 / 75(84)$ & $49 / 58(84)$ & $10 / 75(7)$ \\
\hline Lymphoma & $27 / 33(82)$ & $26 / 30(87)$ & $7 / 32(21)$ \\
\hline Hodgkin & $7 / 9(78)$ & $7 / 9(78)$ & $4 / 9(44)$ \\
\hline Non-Hodgkin & $20 / 24(87)$ & $19 / 21(91)$ & $3 / 24(13)$ \\
\hline Metastatic lymphadenopathy from extrathoracic malignancy & $20 / 25(80)$ & $11 / 15(73)$ & $3 / 25(12)$ \\
\hline Lung cancer & $16 / 16(100)$ & $12 / 12(100)$ & $0 / 16(0)$ \\
\hline NSCLC & $5 / 5(100)$ & $4 / 4(100)$ & $0 / 5(0)$ \\
\hline Small-cell carcinoma of lung & $8 / 8(100)$ & $6 / 6(100)$ & $0 / 8(0)$ \\
\hline Pulmonary neuroendocrine tumor & $3 / 3(100)$ & $2 / 2(100)$ & $0 / 3(0)$ \\
\hline Post-transplant lymphoproliferative disorder ${ }^{\mathrm{a}}$ & $0 / 1(0)$ & $0 / 1(0)$ & $0 / 1(0)$ \\
\hline Benign diagnosis & $240 / 275(87)$ & $155 / 175(88)$ & $20 / 275(7)$ \\
\hline Sarcoidosis & $123 / 149(83)$ & $82 / 94(87)$ & $9 / 149(6)$ \\
\hline Reactive lymphocytosis & $95 / 101(94)$ & $63 / 66(96)$ & $6 / 101(6)$ \\
\hline Necrotizing granuloma including TB, NTM, and fungal infection & $13 / 19(68)$ & $5 / 10(50)$ & $6 / 19(32)$ \\
\hline TB/NTM & $7 / 10(70)$ & $4 / 5(80)$ & $4 / 10(40)$ \\
\hline Fungal & $1 / 3(33)$ & $1 / 2(50)$ & $2 / 3(67)$ \\
\hline Others ${ }^{\mathrm{b}}$ & $6 / 6(100)$ & $5 / 5(100)$ & $0 / 6(0)$ \\
\hline All diagnosis & $300 / 350(86)$ & $233 / 233(67)$ & $32 / 350(9)$ \\
\hline
\end{tabular}

EBUS-TBNA, endobronchial ultrasound-guided transbronchial needle aspiration; ROSE, rapid on-site evaluation; NSCLC, nonsmall cell lung cancer; TB, tuberculosis; NTM, non-tuberculous mycobacterium. ${ }^{a}$ Diagnosis made by transbronchial forceps biopsy during the same procedure. ${ }^{\mathrm{b}}$ Others include bronchogenic cysts $(n=2)$, histiocytosis $(n=1)$, mastocytosis $(n=1)$, amyloidosis $(n=1)$ and silicosis $(n=1)$. 


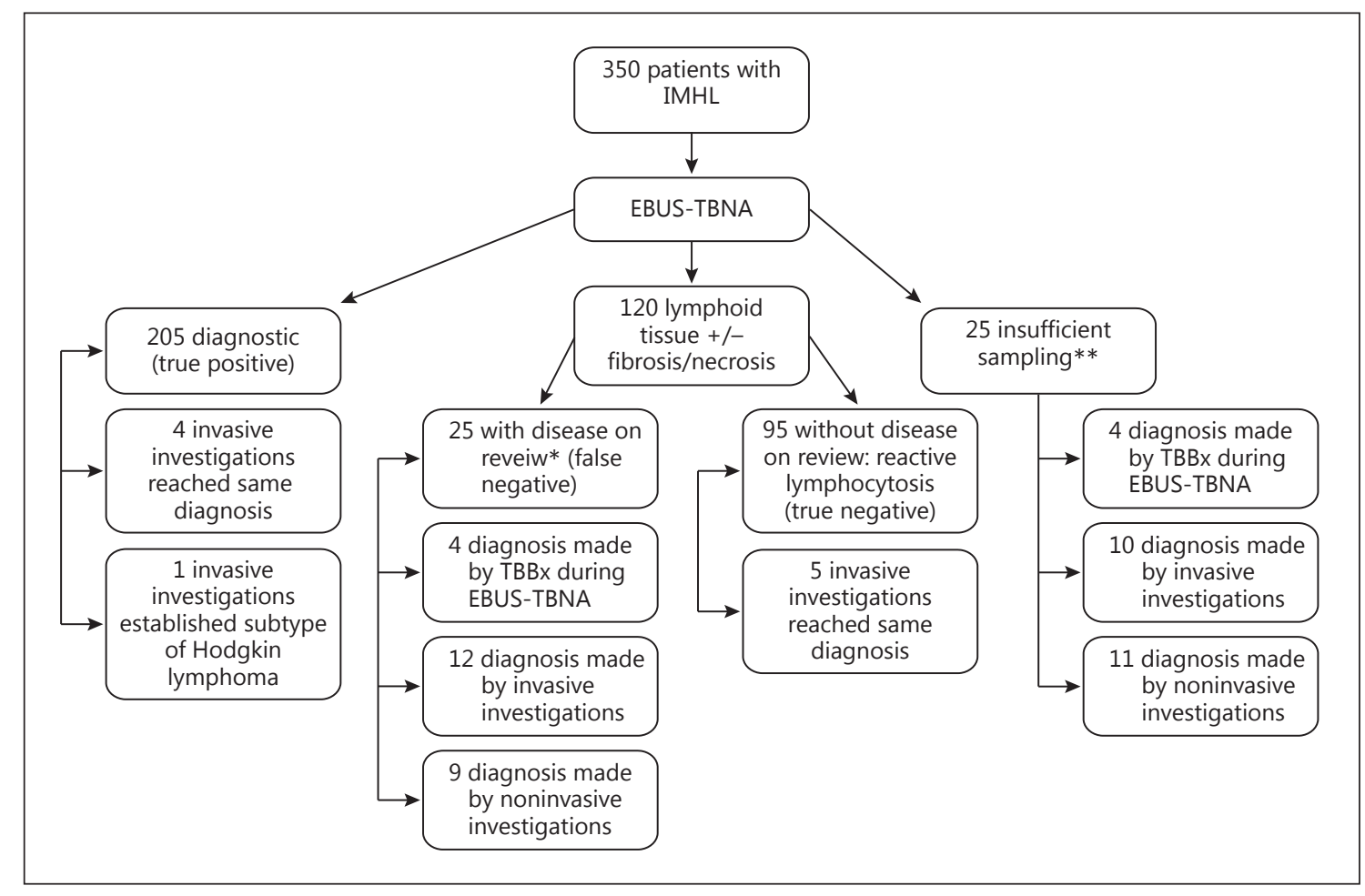

Fig. 2. Subsequent investigations after initial EBUS-TBNA. IHML, isolated mediastinal and hilar lymphadenopathy; EBUS-TBNA, endobronchial ultrasound-guided transbronchial needle aspiration; TBBx, transbronchial biopsy. * False-negative patients include patients with sarcoidosis $(n=14)$, necrotizing granuloma $(n=6)$, lymphoma $(n=4)$, or intrathoracic metastasis from an extrathoracic malignancy $(n=1)$. ** Insufficient sampling patients include patients with reactive lymphocytosis $(n=6)$, sarcoidosis $(n=12)$, intrathoracic metastasis from an extrathoracic malignancy $(n=4)$, lymphoma $(n=1)$, tuberculosis $(n=1)$, or post-transplant lymphoproliferative disease $(n=1)$.

TBNA (82\%): 7 Hodgkin lymphoma and 20 non-Hodgkin lymphoma. Seven additional invasive investigations were performed to obtain diagnosis or confirm subtypes of lymphoma. Fourteen patients had a history of treated lymphoma, and the diagnostic rate by EBUS-TBNA was $93 \%(13 / 14)$. In contrast, $74 \%(14 / 19)$ of the patients with no known history of lymphoma were diagnosed by EBUSTBNA alone.

Intrathoracic lymphadenopathy with known concurrent or preexisting malignancy was found in 114 patients. Out of these patients, 23 patients had a final diagnosis of metastatic lymphadenopathy from extrathoracic malignancy. Two patients were found to have extrathoracic malignancy after being diagnosed with metastatic lymphadenopathy. EBUS-TBNA was diagnostic in 20 patients (80\%).

Mediastinal lymphadenopathy with pulmonary primary malignancy was found in 13 patients. All 8 patients with a final diagnosis of small-cell carcinoma were diagnosed by EBUS-TBNA alone. Similarly, all 5 patients with a final diagnosis of adenocarcinoma favoring pulmonary origin were diagnosed by EBUS-TBNA alone.

Overall, EBUS-TBNA had a sensitivity of $89 \%$, an NPV of $79 \%$ (Fig. 2), and an overall diagnostic rate of $86 \%$. Thirty-two invasive procedures were performed after initial EBUS to further investigate the patients at the discretion of the referring physician.

\section{Discussion}

The study demonstrates the effectiveness of EBUSTBNA in the investigation of patients with IMHL. After initial testing with EBUS-TBNA, diagnostic sensitivity is $89 \%$. With long-term follow-up by chart review, we calculated an NPV of 79\%. Although our diagnostic sensitivity of EBUS-TBNA in patients with IHML reflects that of previous studies $[9,11-13]$, the NPV presented in this study is much higher in comparison with one exception. Similar 
to our study, the study by Evison et al. [11] contains a significant number of patients with reactive lymphadenopathy. In the absence of new pathology or clinical diagnosis on follow-up chart review, patients with initially normal lymphoid tissue based on EBUS-TBNA were categorized as having reactive lymphadenopathy. The inclusion of reactive lymphadenopathy patients as true negatives is the reason for the high NPV in our studies. Although this result does not negate the need for clinical follow-up, it does give the clinician more confidence of a normal lymphoid tissue when performed in a specialized center.

The diagnostic yield of EBUS-TBNA in patients with sarcoidosis is $83 \%$ and it is consistent with a recent metaanalysis by Agarwal et al. [6]. Furthermore, in patients identified with mucosal changes consistent with sarcoidosis, endobronchial biopsy of the mucosa during the same procedure would increase the overall yield to $88 \%$. This data echoes the finding in a study by Goyal et al. [14], where the authors demonstrate that a combination of bronchoscopy-guided biopsy would maximize the yield during a single procedure. Unique to our study is a new insight into the prevalence of sarcoidosis in patients with IHML and concurrent or previously treated malignancy: $24 \%$ (27/115). In a similar study that only included patients with previously treated cancer, Kennedy et al. [15] report an 8\% prevalence of sarcoidosis. The difference in prevalence is attributed to the patients included in the study, while we also recognize that both studies are single centered and prone to referral bias. The clinical implication of the data further supports the clinical diagnosis of "sarcoid reaction to malignancy" [16] and "cancer therapy-induced sarcoidosis" [17]. More importantly, the presented data support the need for tissue confirmation in patients suspected to have higher-stage malignancy or recurrence.

Current data on the diagnosis of lymphoma by EBUSTBNA has shown wide variability [18-21]. Nevertheless, our diagnostic yield is consistent with studies presented by Moonim et al. [20] and Grosu et al. [21], where the diagnostic yield is in the range of mid- $80 \%$. Two potential explanations may account for the differences. First, as described in the Material and Method section, we chose ROSE for patients suspected to have lymphoproliferative disease. In our practice, the clinician informs the attending cytopathologist of the suspected diagnosis. During the procedure, the on-site cytology assessment guided the number of passes and the area in the lymph node to biopsy that yield the best quality specimen. This collaboration ensures that adequate specimens are acquired for both detailed morphologic assessment and ancillary testing required to subtype lymphomas [22]. Secondly, de- spite the wide adoption of fine needle aspiration as the diagnostic tool for lymphoma, societies including the European Society of Medical Oncologist [23] and the National Comprehensive Cancer Network [24] explicitly express preference for excisional biopsies at the time of initial diagnosis. Although this view is not strongly shared by our cytopathologists, the recommendations by these societies may affect the inclination of many pathologists to investigate beyond "suspicious for lymphoma," and attempt specific lymphoma classification.

Although no definitive conclusion can be drawn due to the number of lymphoma patients in this study, our data show a trend towards lower diagnostic sensitivity for Hodgkin disease. Similar observations have been documented $[19,21]$. The difficulty in diagnosing Hodgkin lymphoma can be largely attributed to the frequent paucity of Reed-Sternberg cells in cell block sections precluding the necessary confirmatory immunohistochemical stains [20]. While excisional biopsy by mediastinoscopy would be the usual next step in obtaining diagnosis, the use of a 19-G EBUS-TBNA needle might provide additional cell block material for immunohistochemistry.

The diagnostic yield of EBUS-TBNA in patients with intrathoracic lymphadenopathy and a history of an extrathoracic malignancy is reported to be in the mid-80\% [7, $25,26]$. In our cohort, $80 \%(20 / 25)$ of the patients with a final diagnosis of metastatic intrathoracic lymphadenopathy were diagnosed by EBUS-TBNA alone. Four out of the 5 patients that were non-diagnostic by EBUS-TBNA had insufficient sampling of the targeted lymph nodes, and the remaining patient had an EBUS-TBNA cytopathology report indicating necrosis. Contrary to a previous report of low NPV by EBUS-TBNA in the diagnosis of metastatic intrathoracic lymphadenopathy [7], we documented only one false-negative in this subgroup. While we agree that clinical follow-up with non-invasive imaging is pertinent in patients with a history of extrathoracic malignancy and an EBUS-TBNA report of an alternative diagnosis, further invasive investigation may not be immediately warranted.

Two task force guidelines suggest that the presence of ROSE in EBUS-TBNA procedures reduces the need for additional procedures without affecting the diagnostic yield, procedure time, and complication rate $[27,28]$. However, the referenced EBUS-TBNA studies are mostly based on patients who are clinically suspicious for primary lung cancer $[29,30]$. Our center chose to have ROSE for patients clinically suspected to have lymphoproliferative disease and/or granulomatous disease. The rationale behind the decision was to receive real-time feedback for adequacy of
462

Respiration 2017;94:457-464

DOI: $10.1159 / 000479745$
Tyan et al. 
specimen and appropriation of diagnostic samples for ancillary testing. Our yield in patients diagnosed with lymphoma (87\%) and sarcoidosis (87\%) with ROSE are both in the higher range of what is currently documented in the literature $[6,8]$. This finding highlights a potential association between the utilization of ROSE and higher diagnostic yield within these subgroups. Further studies are required to validate this clinical correlation.

There are limitations to our study. This is a retrospective study that examines the available data in our institution. While recommendations are made by the bronchoscopist upon completion of the procedure, the subsequent investigation is at the discretion of the referring physician. In these circumstances, the patients with an "insufficient sample" from EBUS-TBNA would no doubt trigger further investigations. However, patients with a cytologic diagnosis of benign lymphoid tissue or reactive lymphocytosis have the potential to be underinvestigated, overestimating the NPV and sensitivity. In addition, the study location is not endemic with tuberculosis, and, therefore, the same generalization cannot be made to populations that are.

In conclusion, in this study of IMHL patients and high background prevalence of concurrent and preexisting non-pulmonary malignancy, EBUS-TBNA is a reliable diagnostic first-line investigation with a high NPV.

\section{Acknowledgement}

This study was supported by researchers at the University Health Network. We thank Alexandria Grindlay and Judy McConnell for trial support and Frances Allison for assisting with submission of Ethics Board approval. We are grateful for all the clinical information provided by the patients and referring physicians.

\section{Financial Disclosure and Conflicts of Interest}

The authors declare that they have no conflict of interest.

\section{Author Contributions}

Dr. Tyan had full access to all the data in the study and takes responsibility for the integrity of the data and the accuracy of the data analysis.

Dr. Tyan: contributed to the organization, study design, data collection, statistical analysis, and manuscript writing.

Dr. Machuca: contributed to the study design, data collection, and writing of the manuscript.

Dr. Czarnecka: contributed to data collection and writing of the manuscript.

Dr. Ko: contributed to the interpretation of data and writing of the manuscript.

Dr. Gilda da Cunha: contributed to the interpretation and writing of the manuscript.

Dr. Boerner: contributed to the interpretation of data and writing of the manuscript.

Dr. Pierre: contributed to the data collection and writing of the manuscript.

Dr. Cypel: contributed to the data collection and writing of the manuscript.

Dr. Waddell: contributed to the study design, data collection and writing of the manuscript.

Dr. Darling: contributed to the study design, data collection and writing of the manuscript.

Dr. de Perrot: contributed to the data collection and writing of the manuscript.

Dr. Keshavjee: contributed to the data collection and writing of the manuscript.

Dr. Geddie: contributed to the study design, data interpretation and writing of the manuscript.

Dr. Yasufuku: contributed as the principal investigator for this study to project oversight; in addition, he contributed to the data collection and writing of the manuscript.

\section{References}

1 Kirchner J, Kirchner EM, Goltz JP, Lorenz V-W, Kickuth R: Prevalence of enlarged mediastinal lymph nodes in heavy smokers - a comparative study. Eur Radiol 2011;21:1594-1599.

-2 Souza CA, Müller NL, Lee KS, Johkoh T, Mitsuhiro $\mathrm{H}$, Chong S: Idiopathic interstitial pneumonias: prevalence of mediastinal lymph node enlargement in 206 patients. AJR Am J Roentgenol 2006;186:995-999.

- 3 Joffre F, Remy J, Remy-Jardin M, Chabbert VR, Canevet G, Baixas CC, et al: Mediastinal lymphadenopathy in congestive heart failure: a sequential CT evaluation with clinical and echocardiographic correlations. Eur Radiol 2004;14:881-889.

\footnotetext{
4 Lewin S, Goldberg L, Dec GW: The spectrum of pulmonary abnormalities on computed chest tomographic imaging in patients with advanced heart failure. Am J Cardiol 2000;86: 98-100.

-5 Yasufuku K, Pierre A, Darling G, de Perrot M, Waddell T, Johnston M, et al: A prospective controlled trial of endobronchial ultrasoundguided transbronchial needle aspiration compared with mediastinoscopy for mediastinal lymph node staging of lung cancer. J Thorac Cardiovasc Surg 2011;142:1393-2400.e1.

-6 Agarwal R, Srinivasan A, Aggarwal AN, Gupta D: Efficacy and safety of convex probe EBUS-TBNA in sarcoidosis: a systematic re-
}

view and meta-analysis. Respir Med 2012; 106:883-892.

7 Navani N, Nankivell M, Woolhouse I, Harrison RN, Munavvar M, Oltmanns $\mathrm{U}$, et al: Endobronchial ultrasound-guided transbronchial needle aspiration for the diagnosis of intrathoracic lymphadenopathy in patients with extrathoracic malignancy: a multicenter study. J Thorac Oncol 2011;6:15051509.

8 Rao AK, Ben-Or S, Bowling MR: Bronchoscopic myths and legends transbronchial needle aspiration of mediastinal and hilar lymph nodes in the diagnosis of lymphoma. Clin Pulm Med 2014;21:50-52.
Performance of EBUS-TBNA in

Mediastinal Disease
Respiration 2017;94:457-464 DOI: $10.1159 / 000479745$ 
$\checkmark$ Navani N, Lawrence DR, Kolvekar S, Hayward M, McAsey D, Kocjan G, et al: Endobronchial ultrasound-guided transbronchial needle aspiration prevents mediastinoscopies in the diagnosis of isolated mediastinal lymphadenopathy: a prospective trial. Am J Respir Crit Care Med 2012;186:255-260.

-10 Madan K, Mohan A, Ayub II, Jain D, Hadda V, Khilnani GC, et al: Initial experience with endobronchial ultrasound-guided transbronchial needle aspiration (EBUS-TBNA) from a tuberculosis endemic population. J Bronchology Interv Pulmonol 2014;21:208-214.

$\checkmark 11$ Evison M, Crosbie PAJ, Morris J, Martin J, Barber PV, Booton R: A study of patients with isolated mediastinal and hilar lymphadenopathy undergoing EBUS-TBNA. BMJ Open Respir Res 2014;1:e000040.

-12 Boujaoude Z, Dahdel M, Pratter M, Kass J: Endobronchial ultrasound with transbronchial needle aspiration in the diagnosis of bilateral hilar and mediastinal lymphadenopathy. J Bronchology Interv Pulmonol 2012;19: 19-23.

-13 Yasufuku K, Nakajima T, Fujiwara T, Yoshi no I, Keshavjee S: Utility of endobronchial ultrasound-guided transbronchial needle aspiration in the diagnosis of mediastinal masses of unknown etiology. Ann Thorac Surg 2011;91:831-836.

-14 Goyal A, Gupta D, Agarwal R, Bal A, Nijhawan R, Aggarwal AN: Value of different bronchoscopic sampling techniques in diagnosis of sarcoidosis: a prospective study of $151 \mathrm{pa}-$ tients. J Bronchology Interv Pulmonol 2014; 21:220-226.

$\checkmark 15$ Kennedy MP, Jimenez CA, Mhatre AD, Morice RC, Eapen GA: Clinical implications of granulomatous inflammation detected by endobronchial ultrasound transbronchial needle aspiration in patients with suspected cancer recurrence in the mediastinum. J Cardiothorac Surg 2008;3:8
6 Brincker H: Sarcoid reactions in malignant tumours. Cancer Treat Rev 1986;13:147-156.

17 Umezu H, Chida M, Inoue T, Araki O, Tamura $\mathrm{M}$, Tatewaki M, et al: Sarcoidosis development during induction chemotherapy for lung cancer mimicked progressive disease. Gen Thorac Cardiovasc Surg 2010;58:434437.

18 Iqbal S, DePew ZS, Kurtin PJ, Sykes AMG, Johnson GB, Edell ES, et al: Endobronchial ultrasound and lymphoproliferative disorders: a retrospective study. Ann Thorac Surg 2012; 94:1830-1834.

19 Talebian-Yazdi M, Bartheld von B, Waaijenborg F, Nabes H, Venmans B, Veselic-Charvat M, et al: Endosonography for the diagnosis of malignant lymphoma presenting with mediastinal lymphadenopathy. J Bronchology Interv Pulmonol 2014;21:298-305.

20 Moonim MT, Breen R, Fields PA, Santis G: Diagnosis and subtyping of de novo and relapsed mediastinal lymphomas by endobronchial ultrasound needle aspiration. Am J Respir Crit Care Med 2013;188:1216-1223.

21 Grosu HB, Iliesiu M, Caraway NP, Medeiros LJ, Lei X, Jimenez CA, et al: Endobronchial ultrasound-guided transbronchial needle aspiration for the diagnosis and subtyping of lymphoma. Ann Am Thorac Soc 2015;12: 1336-1344.

-22 Ko HM, da Cunha Santos G, Darling G, Pierre A, Yasufuku K, Boerner SL, et al: Diagnosis and subclassification of lymphomas and nonneoplastic lesions involving mediastinal lymph nodes using endobronchial ultrasound-guided transbronchial needle aspiration. Diagn Cytopathol 2013;41:1023-1030.

23 Tilly H, Vitolo U, Walewski J, da Silva MG, Shpilberg O, Andre M, et al: Diffuse large Bcell lymphoma (DLBCL): ESMO Clinical Practice Guidelines for diagnosis, treatment and follow-up. Ann Oncol 2012;23(suppl 7):vii78-vii82.
24 Zelenetz AD, Abramson JS, Advani RH, Andreadis CB, Byrd JC, Czuczman MS, et al: NCCN Clinical Practice Guidelines in Oncology: non-Hodgkin's lymphomas. J Natl Compr Canc Netw 2010;8:288-334.

25 Song J-U, Park HY, Jeon K, Koh W-J, Suh GY, Chung MP, et al: The role of endobronchial ultrasound-guided transbronchial needle aspiration in the diagnosis of mediastinal and hilar lymph node metastases in patients with extrapulmonary malignancy. Intern Med 2011;50:2525-2532.

-26 Tournoy KG, Govaerts E, Malfait T, Dooms $\mathrm{C}$ : Endobronchial ultrasound-guided transbronchial needle biopsy for M1 staging of extrathoracic malignancies. Ann Oncol 2010; 22:127-131.

27 Wahidi MM, Herth F, Yasufuku K, Shepherd RW, Yarmus L, Chawla M, et al: Technical aspects of endobronchial ultrasound-guided transbronchial needle aspiration. Chest 2016; 149:816-835.

28 van der Heijden EHFM, Casal RF, Trisolini R, Steinfort DP, Hwangbo B, Nakajima T, et al: Guideline for the acquisition and preparation of conventional and endobronchial ultrasound-guided transbronchial needle aspiration specimens for the diagnosis and molecular testing of patients with known or suspected lung cancer. Respiration 2014;88:500-517.

29 Oki M, Saka H, Kitagawa C, Kogure Y, Murata N, Adachi T, et al: Rapid on-site cytologic evaluation during endobronchial ultrasound-guided transbronchial needle aspiration for diagnosing lung cancer: a randomized study. Respiration 2013;85:486-492.

>30 Nakajima T, Yasufuku K, Saegusa F, Fujiwara T, Sakairi Y, Hiroshima K, et al: Rapid on-site cytologic evaluation during endobronchial ultrasound-guided transbronchial needle aspiration for nodal staging in patients with lung cancer. Ann Thorac Surg 2013;95:16951699. 